\title{
Understanding the Impacts of the COVID-19 Pandemic on Public Transportation Travel Patterns in the City of Lisbon
}

\author{
Joao T. Aparicio 1,2,*, Elisabete Arsenio $2, *$ (D) and Rui Henriques $1, *$ (D) \\ 1 INESC-ID, Instituto Superior Técnico, Universidade de Lisboa, 1049-001 Lisbon, Portugal \\ 2 LNEC, Department of Transport, 1700-066 Lisbon, Portugal \\ * Correspondence: joao.aparicio@tecnico.ulisboa.pt (J.T.A.); earsenio@lnec.pt (E.A.); \\ rmch@tecnico.ulisboa.pt (R.H.); Tel.: +351-21-310-0300 (R.H.)
}

Citation: Aparicio, J.T.; Arsenio, E.; Henriques, R. Understanding the Impacts of the COVID-19 Pandemic on Public Transportation Travel Patterns in the City of Lisbon. Sustainability 2021, 13, 8342. https://doi.org/10.3390/su13158342

Academic Editors: José Manuel Vassallo and Juan Gómez

Received: 1 June 2021

Accepted: 7 July 2021

Published: 26 July 2021

Publisher's Note: MDPI stays neutral with regard to jurisdictional claims in published maps and institutional affiliations.

Copyright: (c) 2021 by the authors. Licensee MDPI, Basel, Switzerland. This article is an open access article distributed under the terms and conditions of the Creative Commons Attribution (CC BY) license (https:/ / creativecommons.org/licenses/by/ $4.0 /)$.

\begin{abstract}
The ongoing COVID-19 pandemic is creating disruptive changes in urban mobility that may compromise the sustainability of the public transportation system. As a result, worldwide cities face the need to integrate data from different transportation modes to dynamically respond to changing conditions. This article combines statistical views with machine learning advances to comprehensively explore changing urban mobility dynamics within multimodal public transportation systems from user trip records. In particular, we retrieve discriminative traffic patterns with order-preserving coherence to model disruptions to demand expectations across geographies and show their utility to describe changing mobility dynamics with strict guarantees of statistical significance, interpretability and actionability. This methodology is applied to comprehensively trace the changes to the urban mobility patterns in the Lisbon city brought by the current COVID-19 pandemic. To this end, we consider passenger trip data gathered from the three major public transportation modes: subway, bus, and tramways. The gathered results comprehensively reveal novel travel patterns within the city, such as imbalanced demand distribution towards the city peripheries, going far beyond simplistic localized changes to the magnitude of traffic demand. This work offers a novel methodological contribution with a solid statistical ground for the spatiotemporal assessment of actionable mobility changes and provides essential insights for other cities and public transport operators facing mobility challenges alike.
\end{abstract}

Keywords: public transportation; multimodality; COVID-19; order-preserving traffic dynamics; discriminative pattern mining; sustainable mobility

\section{Introduction}

Worldwide, cities face pressures to dynamically adapt their public transportation systems to better respond to the increasingly complex changes in urban mobility. Pandemicevoked changes are driven by shifts in travel patterns, increased traffic demand variability, the need to enforce safety norms of social distancing and hygiene, and the rising advocacy towards active modes of transportation [1]. In a disruptive and evolving socioeconomic context, such as a pandemic one, the value of static studies with fixed temporal spans is thus of limited relevance as their findings can easily become obsolete [2]. Instead, the presence of an enhanced methodology for the dynamic and comprehensive tracing of actionable changes to the urban mobility dynamics is necessary so that the finding can be periodically updated in the presence of incoming multimodal traffic data [3]. Still, the research literature on the dynamic assessment of the impacts of COVID-19 on public transport use is limited [4].

This work provides a comprehensive and actionable description of non-trivial traffic demand changes within a multimodal public transportation system between two reference periods from individual trip data. The city of Lisbon in Portugal is used as the reference study case to this end. Complete individual trip record data along the public transportation system from 2019 and 2020 is considered in this study. Public transportation in Lisbon 
operates under an integrated fare collection, offering the unprecedented possibility to trace the movements of each user along multiple modes within the public transportation system. In particular, we consider trip record data gathered from the three major public transportation modes in Lisbon: subway or underground, bus, and tramways.

The discovery of changing mobility patterns in urban centers is hindered by four major challenges [5,6]. First, trip record data is inherently multimodal and shows spatiotemporal stochasticities. The rich modal, geographical, and calendrical content of trip record data should be properly explored. Second, massive timestamped validations are produced at subway stations, buses, and trams around the city. Over 50 million trips are recorded in Lisbon per month. Analyzing such massive data comes with strict scalability requirements for the pursued processing and learning algorithms. Third, there is the need to go beyond trivial changes in demand into more informative views that can account for changes in mode preference as well as emerging traffic origin-destination flows throughout the city. Fourth, there is the need to guarantee the statistical significance, actionability, interpretability, and navigability of the changing urban mobility patterns. This work applies a novel methodology to answer the above challenges, characterizing mobility dynamics in urban centers from user trip data, combining both statistical principles with advanced pattern mining principles based on order-preserving biclustering searches.

The gathered results comprehensively map statistically significant changed travel patterns within the Lisbon city, going far beyond simplistic localized changes to the magnitude of traffic demand per geography. This research offers a statistical ground for the spatiotemporal assessment of actionable mobility changes and provides essential essential insights for other cities and public transport operators facing mobility challenges alike.

This work is anchored in the pioneer research and innovation project ILU, a project that joins the Lisbon city Council and national research institutes, bridging the ongoing research on urban mobility with recent advances from artificial intelligence.

\section{Background}

Automated fare collection (AFC) systems produce individual trip records in public transportation, generally consisting of smart card validations from users at stations or vehicles. For each card validation, an individual trip record is issued with the passenger identifier, timestamp, boarding or alighting location and, for validations inside vehicles, additional details pertaining to the vehicle and route. In cities, such as Lisbon, the ticketing systems of public carriers are consolidated, offering the possibility to trace multimodal user movements along the public transportation network.

Individual trip record data are generally subjected to statistical exploration, visual inspection, and often mapped into spatiotemporal data structures more conducive to subsequent traffic demand analysis. Three major structural representations of trip record data can be found: georeferenced time series of traffic demand at different locations and routes; end-to-end origin-destination (OD) data mapped from paired entry-and-exit card validations of users along the public transport network; and raw trip/event data. Given a specific traffic data structure, descriptive tasks generally aim at extracting statistically significant patterns or to generatively model traffic dynamics; predictive tasks are considered when forecasting upcoming traffic dynamics or to discriminate particular traffic conditions of interest; and prescriptive tasks rely on optimization principles over the described/predicted traffic dynamics to place mobility recommendations. Computational approaches for the analysis of traffic demand and OD series generally rely on classic statistical principles including decomposition, auto-regression, differencing and exponential smoothing operations - and on advances from machine learning - distance-based approaches reliant on series similarities (motif analysis, lazy learning, barycenter computation) [7] and recurrent neural network approaches for the autonomous learning of complex temporal associations. Complementary approaches have been proposed for raw trip record data analysis, including distance-based and generative approaches from event-sets [8], episode mining [9], and dedicated neural processing architectures [10]. More frequently, raw trip 
data analysis resorts to the introduced approaches for demand/OD traffic series under specific spatiotemporal aggregation criteria.

Given a specific spatiotemporal data structure, a pattern is a set of spatially correlated, coherently changing observations along time [11]. Illustrating, periodic patterns or urban traffic describe recurrent demand over regular time intervals at certain locations or origindestination flows. For a given pattern solution, different criteria of interest can be measured: (i) pattern support, the number of observations satisfying the pattern; (ii) pattern length, the multivariate order and spatiotemporal extension of the given pattern; and (iii) pattern strength, including confidence, lift and interestingness, defining the association strength among the elements composing a pattern.

Specific contributions within the spatiotemporal pattern mining field aim at describing changing dynamics. Emerging Patterns (EPs) were firstly introduced by Dong et al. [12] in the context of multivariate observations collected from two periods/datasets. An emerging pattern was in this context defined as a multivariate pattern whose support suffered a significant change between the two given periods. Neves et al. [5] extended this early notion of emerging pattern to encompass an arbitrary number of time periods and to further incorporate spatial information. In the context of this work, an emerging pattern is defined as a set of spatially correlated observations whose values satisfy specific growth, fitness and support criteria along time. The growth criterion defines the rate at which observations change along time. Given a specific growth rate, the fitness (quality) criterion defines how well observations follow (deviate) from the given expectations. Finally, support criterion defines the number of observations (temporal extent) satisfying the given growth and accuracy criteria. Emerging patterns not satisfying specific growth, quality and support criteria may be spurious and should therefore be discarded. Paradigmatic examples include patterns without clear trends or guarantees of fitness quality due fitting errors and/or lack of support for sound statistical testing.

Despite the relevance of these previous pattern-centric studies, they are arguably insufficient to assess changing traffic patterns along a pandemic context. Given the high extent of mobility changes observed throughout pandemic contexts, there is the need to deal with arbitrarily-high volume of patterns and go beyond trivial views focused on how demand is changing at specific locations, into more actionable views able to capture changing travel patterns throughout a urban centre. In this context, and in addition to the introduced growth, quality and support criteria, emerging patterns should further satisfy the following properties of interest: (i) non-triviality (novelty); (ii) actionability (ability to support real-world decisions and reveal relevant knowledge); (iii) robustness (bounded noise tolerance); (iv) statistical significance (excluded spurious patterns occurring by chance); (v) interpretability; (vi) coverage (complete solutions spanning different geographies and time periods); and (vii) efficiency of the pattern retrieval process.

\section{Related Work}

The discovery of actionable spatiotemporal mobility patterns has received particular attention in recent years with the increased availability of urban data, advances on spatiotemporal data analysis, and global pressure towards sustainability [13-15]. Classic approaches make use of statistics, parametric models and visualization principles to understand spatiotemporal traffic dynamics, with particular focus on highlighting discrepancies between origin-destination matrices $[15,16]$ and establishing views on changing traffic flows [17-19]. Clustering has been also applied to identify geographies with correlated traffic demand [20], detect vulnerabilities along a transport network [21], and complementary spatial associations assessed against external factors of influence [22]. Classic pattern mining algorithms have been also successfully extended to detect trajectory patterns [23], patterns of daily congestion and spatial propagation [24,25], amongst other patterns of urban mobility [26]. Neves et al. [11] proposed the combined use of spatiotemporal data transformations and biclustering to comprehensively find congestion patterns from heterogeneous sources of road traffic data. Despite their relevance, their application for assessing 
significant mobility changes along a pandemic context requires the visual inspection of the differences between the solutions derived from the multiple periods of interest.

Recent published studies have offered a description of the effects of the COVID-19 pandemic in the urban mobility dynamics [27-29]. Most studies within this context focus on road traffic, identifying general trends observed before versus during quarantine $[27,28]$. Complementarily, some studies assess the pandemic effects on public transportation. Campisi et al. [30] offer a statistical analysis of post-pandemic mobility needs using comprehensive questionnaire data on the Sicily public transportation. The results capture the changes in perceptions of residents regarding transportation, including the importance of remote work and active modes of transportation (e.g., cycling). However, the gathered results are not anchored in the actual patterns from available traffic data. Similarly, Przybylowski et al. [31] rely on questionnaire data to assess the impact of the COVID-19 pandemic on public transport users in Gdansk, Poland. Sharifi and Khavarian-Garmsird provide a qualitative assessment on the impacts of COVID-19 in the air and water quality across cities during lockdown periods, and offer recommendations related to the socioeconomic factors, urban management, governance, transportation and urban design that can be used for post-COVID urban planning and design. Tamagusko and Adelino [32] studied changing traffic dynamics in Portugal during the starting months of the pandemic, measuring drops in public transport demand. This study also establishes a relationship between re-transmission rates and the enforced safety measures put in place by the Portuguese government. Despite its relevance, the study does not explore changing travel patterns.

In contrast with frequent, periodic or anomalous patterns, changing patterns can be dynamically discovered to reveal the impacts of a pandemic context on urban traffic dynamics. The discovery of changing patterns can be traced back to two different research streams. In time series data analysis, the discovery of emerging behaviors generally corresponds to the modeling of both linear and non-linear trends within a time series [33]. Changing behaviors are generally approximated using regressive or auto-regressive models, including regime switching models and neural network models, approximated on the original time series or on a decomposed series after removing seasonal and cyclical components [34]. In the pattern mining field, emerging behaviors were in 1999 coupled with the pattern concept, implying the satisfaction of statistical frequency criteria. An emerging pattern (EP), as firstly introduced by Dong et al. [12], is a set of data instances whose characteristics entail significant changes between two (or more) periods. This original notion of EPs has been extended and mostly applied in different domains [35,36]. Novak et al. [37] consistently combined principles of contrast set mining, emerging pattern mining, and subgroup discovery with the aim of discovering supervised temporal rules. Chen et al. [38] propose association rule discovery along different time periods. They extend the early Song et al. [39] concepts towards emerging, unexpected and added rules and propose corresponding evaluation measures of growth, difference, and modified difference. In the urban mobility domain, Neves et al. [5] proposed principles for the linear time discovery of emerging and abrupt changes in road traffic along a specific interval.

Alternative time-changing patterns have been proposed for different data structures, including three-dimensional data via triclustering [40]; collections of events using both generative and deterministic approaches [8,41]; as well as streaming data [42] by combining evolutionary algorithms with batch strategies.

Despite the relevance of the surveyed studies, their application for the comprehensive discovery of changing traffic dynamics within a multimodal public transportation system is still generally hampered by some of the following four major challenges:

- the need to go beyond trivial views on how demand is changing at specific locations/routes, and unravel the newly formed circulation dynamics within a city;

- the need to address the high volume of patterns in contexts where urban mobility entails large modifications;

- the need to prioritize changing traffic dynamics in accordance with their discriminative power; and 
- the need to guarantee the statistical significance, interpretability and actionability of the found patterns.

In recent years, a clearer understanding of the synergies between biclustering and pattern mining paved the rise of a new class of algorithms, generally referred to as patternbased biclustering algorithms [43]. In 2021, biclustering found its primary application over intelligent transportation systems [11], allowing the comprehensive discovery of (de)congestion road traffic patterns from stationary and mobile sensor data. Patternbased biclustering algorithms are inherently prepared to efficiently find exhaustive sets of biclusters and offer the unprecedented possibility to affect their structure, coherence and quality [44]. This behavior explains why this class of biclustering algorithms is receiving increasing attention in recent years [43]. BicPAMS (Biclustering based on PAttern Mining Software) consistently combines these state-of-the-art contributions on patternbased biclustering [45].

Despite the relevance of pattern-based biclustering for pattern discovery, they have not been extended for exploring actionable changes in demand from individual trip record data. In the context of our work, BicPAMS is applied with an order-preserving assumption [46] and extended with discriminative power to find changing mobility dynamics over the denormalized traffic data. The statistical frame placed by pattern-based biclustering algorithms in BicPAMS [47] guarantee that the found changing mobility dynamics further satisfy specific criteria of statistical significance and actionability. An actionable pattern is one having a practical value to acquire novel knowledge, aid decision making, or support mobility reforms.

\section{Materials and Methods}

\subsection{Data}

The target individual trip record data were made available by the two major carriers in the Lisbon metropolitan area, CARRIS (the tramway and major bus operator) and METRO (the subway operator). Individual trips correspond to smart card validations at METRO stations and CARRIS buses and tramways, monitored through an integrated fare collection system. In this study we consider all the individual trips recorded throughout a typical pre-pandemic month, October 2019, and a post-pandemic month, May 2020. The Lisbon city was in strict quarantine throughout all days of May of 2020, with changes in the applied quarantine restrictions at two moments, May 2nd and 18th. Along October and May periods, a total of 38,845,645 and 14,867,335 trips were observed at the METRO and CARRIS networks, respectively. An illustrative set of anonymized raw trip records from CARRIS is provided in Table 1.

Table 1. Sample of smart card validation data.

\begin{tabular}{|c|c|c|c|c|c|c|c|c|c|c|c|c|}
\hline Date & Fleet Number & Route Number & Variant & Plate Number & Trip Number & Direction & Stop Number & Card ID (Anonymized) & Type of Title & Title Code & Stop Identifier & Stop Name \\
\hline 24/10/2019 10:03:50 & 201 & $76 \mathrm{~B}$ & 0 & 1 & 6 & CIRC & 7 & 321 & Viagem $\mathrm{CA} / \mathrm{ML}$ & 3032779 & 100318 & R. Cruzeiro /Tv. Pardal \\
\hline 23/10/2019 12:52:06 & 201 & 734 & 8 & 1 & 13 & DESC & 17 & 789 & Viagem CA/ML & 3032779 & 816 & Martim Moniz \\
\hline 24/10/2019 15:49:36 & 201 & $76 \mathrm{~B}$ & 0 & 1 & 16 & CIRC & 3 & 987 & Viagem CA/ML & 3032779 & 13401 & Boa Hora \\
\hline
\end{tabular}

\subsection{Statistical Exploration}

Varying spatiotemporal criteria was considered to identify statistically significant changes in traffic demand within the public transportations system. Spatially, we consider both coarser geographies given by well-established zoning criteria and groups of routes, as well as finer spatial criteria given by stations and routes. Geographical information pertaining to the major city routes vulnerable to congestions is further available. Temporally, we consider different calendrical conditions (weekdays, weekends, day of week), and consider typical weekdays (Tuesdays, Wednesdays and Thursdays) as the default calendar for the conducted statistical analyzes. To assess traffic demand levels, hourly aggregations of trip records are provided by default. A graphical interface with spatiotemporal navigation facilities was further developed for the visualization of demand changes along the Lisbon city for two parameterizable periods of interest. 


\subsection{Discovery of Discriminative Patterns of Changing Traffic Dynamics}

To address the limitations of the surveyed approaches for traffic pattern discovery, we propose the combined use of spatiotemporal data transformations and biclustering to comprehensively find actionable patterns of traffic demand changes from individual trip record data distributed along two periods of interest. In contrast with clustering, biclustering-the discovery of subspaces within real-valued data-provides the possibility to search for traffic patterns across geographies, offering modular views.

Definition 1. Let $\mathcal{S}$ be the set of stations and geographies of interest; $\delta$ be a time interval of interest within a day; and $\tau_{s_{i}}$ be the demand observed for a station, $s_{i} \in \mathcal{S}$, along a $\delta$ interval, generally corresponding to the amount of entry or/and exit card validations. An order of stations, $S \subseteq \mathcal{S}$, is a permutation of stations in accordance with their demand for a given time interval $\delta$, i.e., $\pi_{S}=\tau_{s_{i_{1}}} \leq \tau_{s_{i_{2}}} \leq . . \leq \tau_{s_{i_{|S|} \mid}}$ such that $s_{i_{j}} \in S$ and $j=1 . .|S|$.

Definition 2. Given a set of day instances $\left\{d_{1}, . ., d_{n}\right\}$, set of stations $S$, and time interval $\delta$; an order-preserving pattern is a frequent ordering of stations, $\pi_{S}$, where the frequency should be sufficient to guarantee that the association is statistically significant, thus deviating from null expectations.

Given two reference time periods, $\Delta_{1}$ and $\Delta_{2}$, each with a set of day instances; a discriminative order-preserving pattern corresponds to either an order-preserving pattern in $\Delta_{1}$ that is frequently disrupted in $\Delta_{2}$, denoted $\pi_{S \mid \Delta_{1}}$, or an order-preserving pattern in $\Delta_{1}$ that is frequently disrupted in $\Delta_{2}$, denoted $\pi_{S \mid \Delta_{2}}$.

A changing mobility dynamic is seen as a disrupted ordering of traffic demand among stations or geographies. In other words, changing dynamics occur when order-preserving associations are significantly altered between two time periods. A changing mobility dynamic can be given by a discriminative order-preserving pattern of traffic demand in accordance to Definition 2.

Figure 1 instantiates some of the concepts introduced in Definitions 1 and 2. In this illustrative scenario, we consider the analysis of demand along a set of days from two reference periods, $\Delta_{2019}$ and $\Delta_{2020}$. We observe the formation of two order-preserving patterns, $\pi_{1}=\pi_{\left\{s_{4}, s_{5}, s_{6}\right\} \mid \Delta_{1}}$ and $\pi_{2}=\pi_{\left\{s_{6}, s_{7}, s_{8}\right\} \mid \Delta_{2}}$. While the order-preserving pattern $\pi_{2}$ is discriminative, the order-preserving pattern $\pi_{1}$ is not discriminative as it is not frequently disrupted in $\Delta_{2}$. Further details associated with the discovery process and the computation of the discriminative power and statistical significance of the patterns are introduced later.
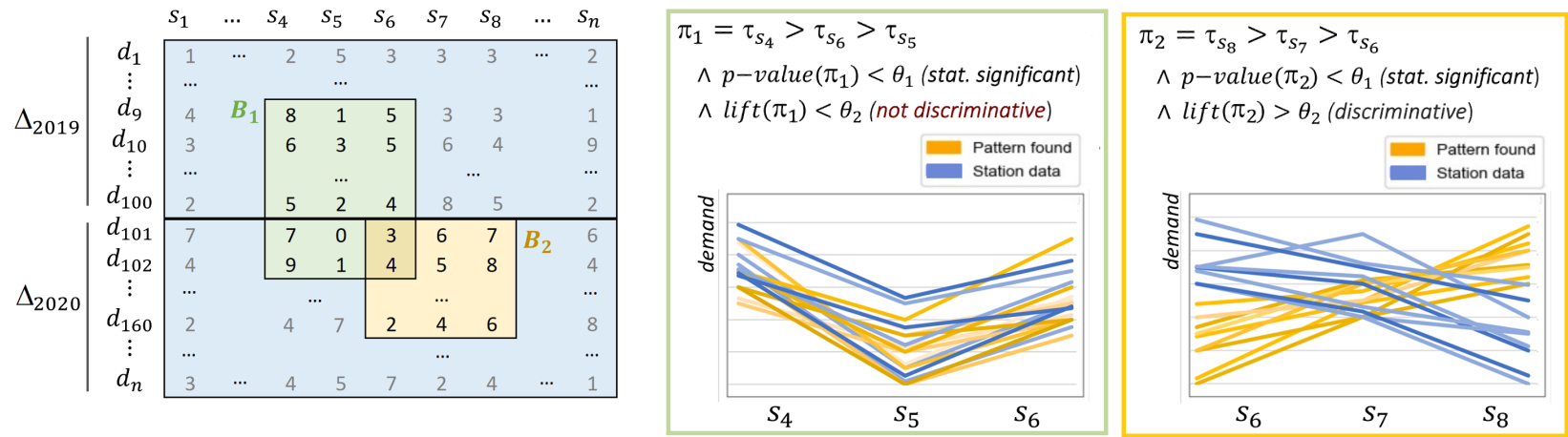

Figure 1. Discovery of discriminative traffic patterns: two illustrative order-preserving demand patterns, $\pi_{1}$ and $\pi_{2}$, both statistically significance yet only one yielding discriminative power.

The intuition behind the target pattern discovery process is to comprehensively identify changes in the orders of demand among stations using two reference periods during particular times of the day (e.g., peak hours). The focus on disrupted order-preserving patterns is essential to guarantee their actionability. Understandably, an alternative and 
simplistic focus on changes to demand levels would lead to large volumes of trivial patterns informing the user of general decreases in demand throughout pandemic times, with poor translation into operational and tactical mobility reforms.

The methodological approach for mining the target discriminative patterns of orderpreserving demand will be introduced along two major steps:

1. data mappings necessary to solve the tackled problem using biclustering tasks;

2. application of biclustering algorithm with order-preserving coherence and guarantees of discriminative power;

3. retrieval and postprocessing of public traffic patterns from biclustering solutions.

\subsubsection{Data Mappings}

The first step of the discovery process of changing mobility dynamics is to fix spatial and temporal constraints, including the target geographies $\mathcal{S}$, time periods $\delta$, and weekday annotations for the composition of data instances. As default, the discovery process considers all available geographies of the search space at their finest level (i.e., stations) and uses typical weekdays as the reference calendar to produce comparable data instances. In addition, the time intervals of interest (e.g., hour, on/off-peak intervals) can be optionally specified to guide road traffic data aggregation. By default, daily demand is considered.

Once these constraints are fixed, data mappings are applied to transform the original spatiotemporal data into a tabular data structure, more conducive to the subsequent pattern mining task. In the target structure, each observation/row represents a day; each variable/column corresponds to a network station or geography (set of stations); and the observed values correspond to the aggregate demand on a given day and station under a specific calendric constraint.

\subsubsection{Discriminative Order-Preserving Biclustering}

Under the previous mappings, traffic data still preserves their spatiotemporal content, yet denormalized within a tabular data structure, turning it a candidate for the application of biclustering. Biclustering aims at finding subsets of observations with values correlated on a subset of variables.

Definition 3. Given a traffic dataset defined by a set of day instances, $\mathcal{D}=\left\{d_{1}, . ., d_{N}\right\}$, stations/geographies $\mathcal{S}=\left\{s_{1}, . ., s_{M}\right\}$, and elements $a_{i j} \in \mathbb{R}$ measuring the demand observed in $d_{i}$ and station $s_{j}$ over a given time interval of the day $\delta$ :

- $\quad$ A bicluster $B=(D, S)$ is a $n \times m$ subspace, where $D=\left(d_{i_{1}}, . ., d_{i_{n}}\right) \subset \mathcal{D}$ is a subset of days and $S=\left(s_{j_{1}}, . ., s_{j_{m}}\right) \subset \mathcal{S}$ is a subset of stations or geographies;

- The biclustering task aims at identifying a set of biclusters $\mathcal{B}=\left\{B_{1}, . ., B_{s}\right\}$ such that each bicluster $B_{k}=\left(D_{k}, S_{k}\right)$ satisfies specific criteria of homogeneity and statistical significance.

Homogeneity criteria are commonly guaranteed through the use of a merit function. Merit functions, such as the variance of the values in a bicluster [48], are typically applied to guide the formation of biclusters in greedy and exhaustive searches. Statistical significance criteria can be further placed to guarantee that the retrieved biclusters cannot occur by chance, i.e., their occurrence deviates from expectations.

Definition 4. Elements in a bicluster $a_{i j} \in(D, S)$ have coherence iff $a_{i j}=c_{j}+\gamma_{i}+\eta_{i j}$, where $c_{j}$ is the expected demand for station $d_{j}, \gamma_{i}$ is the adjustment for day $d_{i}$, and $\eta_{i j}$ is the noise factor.

A bicluster is said to be constant when $\gamma_{i}=0$. A bicluster, $(D, S)$, is order-preserving iff the values for each day in $D$ induce the same linear ordering $\pi$ along the set of stations in $S$.

The constant correlation assumption suffers from a problem: two random days need to show identical demand in order to count as supporting observations of a bicluster. However, demand is inherently stochastic, showing high variability, particularly in a pandemic context. In this context, order-preserving patterns are pursued to guarantee a 
greater robustness to traffic variability, while still guaranteeing the coherence of the target traffic patterns. According to Definitions 2 and 4, order-preserving patterns correspond to frequent orders of demand among a set of stations for a given time interval (e.g., peak hours).

BicPAMS is the suggested biclustering search to the discovery of order-preserving patterns [45]. As discussed with greater detail in Section 3, BicPAMS is inherently ability to retrieve patterns with easily parameterized homogeneity criterion and strict guarantees of optimality and statistical significance $[45,47]$.

To guarantee that the found patterns are discriminative of a specific period, the biclustering search was extended to guarantee that the observed orders yield discriminative power against the period class. Illustrating, given two stations, $s_{A}$ and $s_{B}$, if one station shows consistently higher demand on a period $\Delta_{1}$ and consistently lower demand on $\Delta_{2}$, the corresponding order-preserving patterns, $\pi_{\left\{s_{A}, s_{B}\right\} \mid \Delta_{1}}:\left(\tau_{s_{A}}>\tau_{s_{B}}\right) \Rightarrow \Delta_{1}$ and $\pi_{\left\{s_{A}, s_{B}\right\} \mid \Delta_{2}}:\left(\tau_{s_{B}}>\tau_{s_{A}}\right) \Rightarrow \Delta_{2}$, are discriminative.

To this end, the lift of the candidate association rules,

$$
\operatorname{lift}\left(\pi_{S} \Rightarrow \Delta_{t}\right)=\frac{P\left(\pi_{S \mid \Delta_{t}}\right)}{P\left(\pi_{S}\right) \times P\left(\Delta_{t}\right)},
$$

is assessed in order to guarantee the formation of candidate patterns within the biclustering search is restricted to patterns with lift above a given threshold $\theta$. On one hand, the higher the minimum lift, the higher is the discriminative power of the retrieved order-preserving patterns, i.e., only the more accentuated differences on the demand orderings between time periods $\Delta_{1}$ and $\Delta_{2}$ are returned. On the other hand, lower lifts (yet still considerably above 1) can be useful to guarantee a more comprehensive view of the disrupted orders, even when they occur at a looser discriminative power. From empirical evidence, $\theta=1.5$ is suggested to extract order-preserving patterns with solid discriminative power without incurring in loss of potentially relevant patterns.

\section{Results: Lisbon's Case Study}

The gathered results collected from the application of the proposed methodology to individual trip record data collected from the public transportation system in the Lisbon metropolitan area in pre- and post-pandemic periods are presented in three steps. First, we provide an initial exploration of major differences on the traffic demand. Second, we assess less-trivial changing mobility dynamics using the introduced methodology for mining order-preserving traffic patterns using discriminative biclustering. Third, we offer a more focused discussion on the observed traffic demand differences along the major road routes of the city.

The software for the visual exploration of statistically significant differences (Section 4.2) was developed in Python, and the pattern discovery process (Section 4.3) was implemented in Java as an extension of the BicPAMS software. Both of these facilities are provided within the ILU APP, a recommendation system currently in use by the Lisbon city Council and the public carriers. Experiments were run on Intel Xeon CPU E3-1230 v6 @ 3.50GHz with 16GB RAM.

\subsection{Highlighted Pandemic-Driven Changes in Traffic Demand}

Figure 2 geographically displays the demand at subway stations (transparent circles) and bus-tramway stations (opaque circles) before the pandemic (red-yellow coloring) and during the pandemic (light-dark blue coloring). A general decrease in passengers' demand is observed across METRO (subway) and CARRIS (bus-tramway) networks across the Lisbon metropolitan area. The contraction on the METRO demand per station (red to light-blue circle ratio) is considerably higher than the observed contraction on the CARRIS demand per stop (yellow to dark-blue circle ration). With greater detail, we can observe that the demand across commuting routes leading to Amadora and Odivelas (outside the Lisbon area) was less impacted than the ones leading to Parque das Nações (north-eastern 
side of the city of Lisbon), consistently across the two modes of transport. On the south waterfront zone (Cais do Sodré to Algés) we observe a clear decrease in the demand at CARRIS stops. According to the gross reported income per tax household [49], Amadora and Odivelas, reporting on average 18,157.00 and 19,100.00 euros respectively, have a lower income than the Lisbon municipality (city center), reporting 25,548.00 euros. Generally, the observed degree of demand changes appears to be also correlated with the average land cost near the stations, which serves as a proxy for the household income and working roles that require circulation along the city. Demand changes have a lower magnitude in peripheral stations and in zones with lower income. To further understand the causes of this trend further studies are necessary.

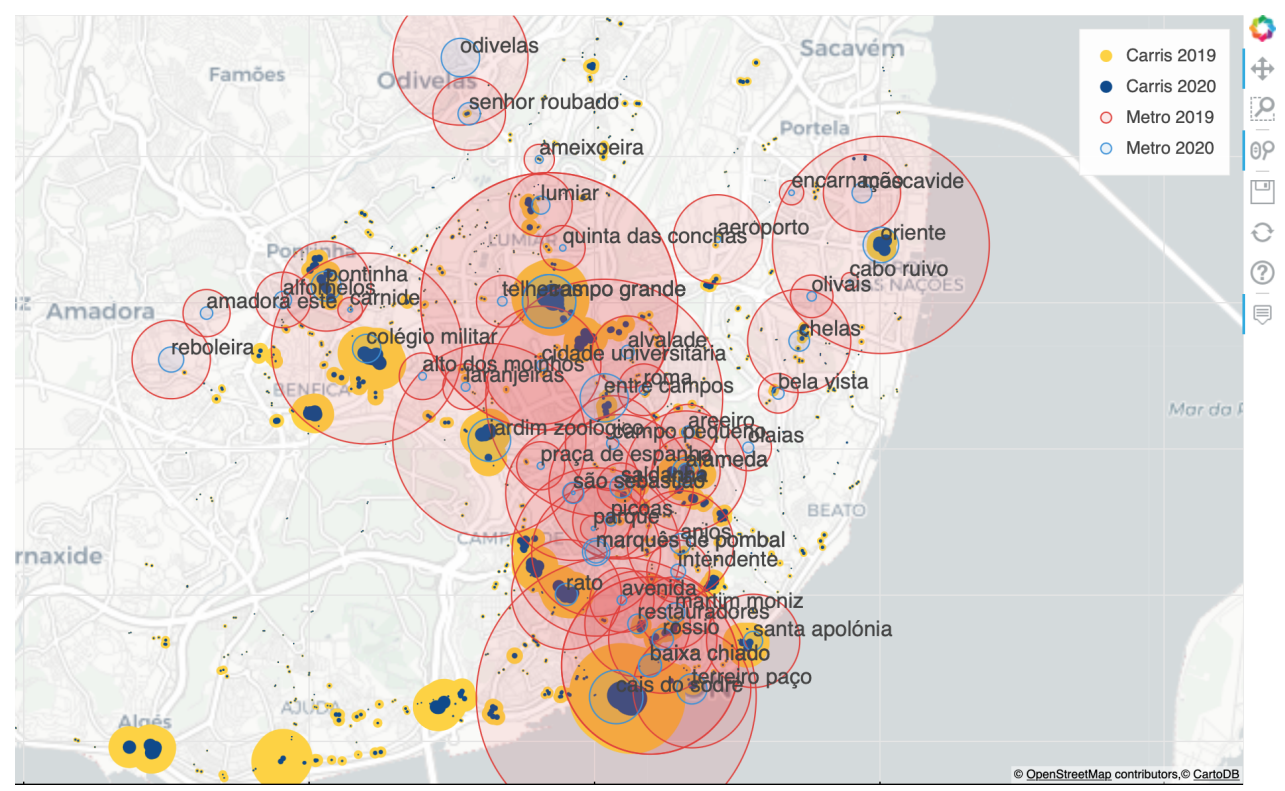

Figure 2. City view of demand variations (2019 vs. 2020) along METRO and CARRIS stations.

Figure 3 provides a coarser-grained view of the daily changes in demand, and the associated variability, across the major lines of the subway network and the major clusters of buses. This analysis underlines the statistically significant nature of the observed differences, and further shows the routes along the city that were subjected to a higher contraction in demand. Generally, the higher the number of stations in the city periphery, the lower the contraction.
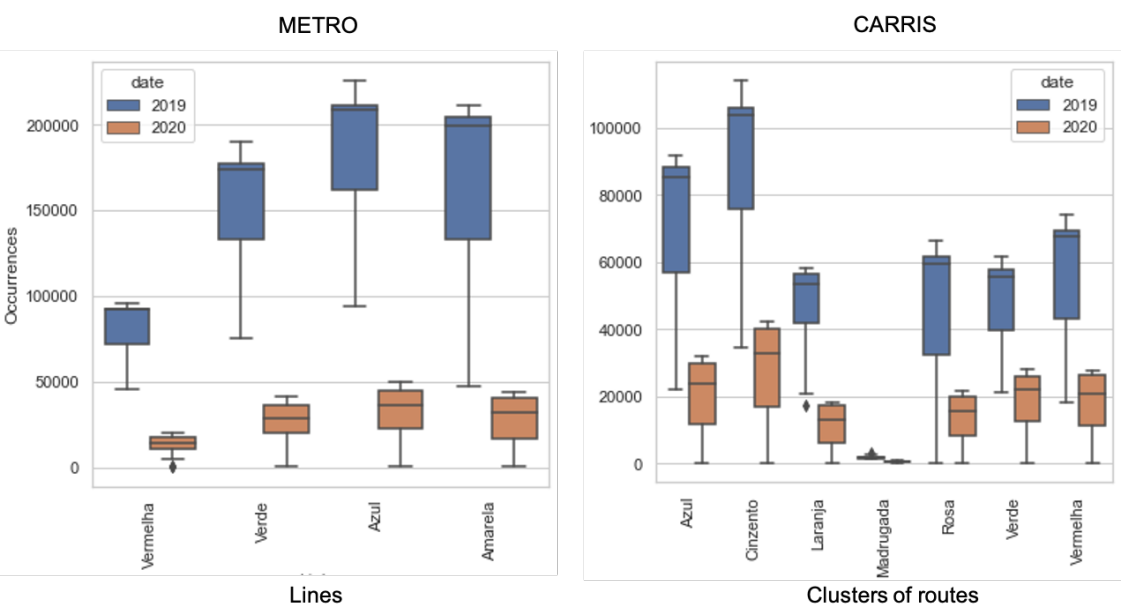

Figure 3. Daily smart card entry validations along the four lines of the METRO network and the seven clusters of routes of the CARRIS network for 2019 versus 2020. 
To clearly assess differences on the degree of demand change within the METRO network, we plotted the demand over the 20 stations with the highest change (left stations in Figure 4) and the 20 stations with lowest change (on the right) across the two time periods. The difference within this mean of transportation is a staggering order of magnitude in all stations. Although the mandatory quarantine was already lifted by May 2020, this result reveals the conservative usage of the public transport propelled by safety and fear considerations. Demand consistently decreased between approximately ten to thirty percent points. The three stations suffering highest demand contraction are: Aeroporto (Lisbon airport), Cidade Universitária (University of Lisbon) and Saldanha (commercial, business and service district in central Lisbon). With less than ten percent of their demand from the previous year, Aeroporto had the most noticeable change due to air travel restrictions held during the reference pandemic period. Cidade Universitária also had a considerable change in demand. This station serves many students as it is one of the main hubs for students to reach several university campi. Saldanha is a working pole, with a high concentration of large business offices. The Saldanha station similarly suffered a significant change in demand due to remote working enforcement rules by the Portuguese government.

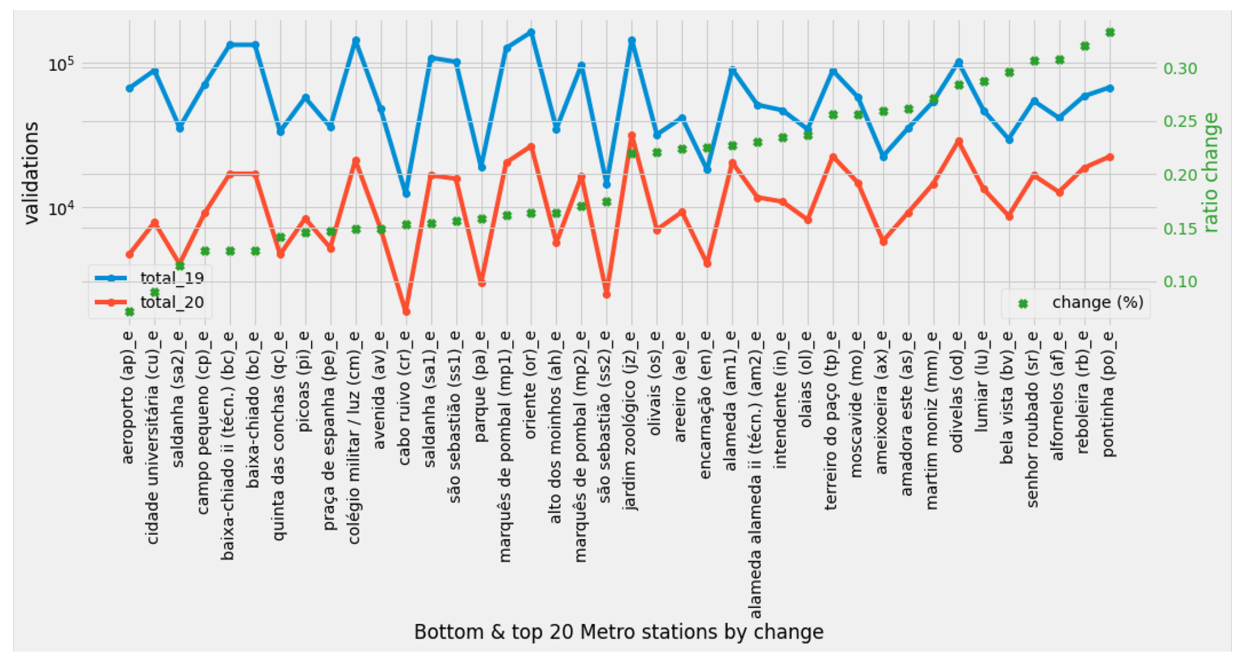

Figure 4. Demand for METRO stations in 2019 versus 2020 and ratio, top and bottom 20 stations.

The changes in demand observed along the CARRIS bus-tramway network reveals a considerably different reality (see Figure 5). For the stations with top differences in demand, the variation yields two orders of magnitude, while some stations did not witness significant changes in demand. In fact, the six stops yielding the least decrease actually show increased levels of demand for 2020. A higher demand in a particular stop may be due to a lack of options on other means of transportation. The R. Castelo Branco Saraiva stop recorded more than 2.5 times the number of validations in 2020 than in 2019. The closest subway station from this stop, Anjos station, suffered a considerable decrease, yielding less than $20 \%$ of the demand observed in the previous year.

Figures 6 and 7 assess the average hourly demand for METRO and CARRIS networks throughout typical working days (top) and weekends (bottom) for the stations with the highest and lowest demand variation from 2019 to 2020. The presented heatmaps have a logarithmic demand scale to foster interpretability and softening differences of high magnitude. 


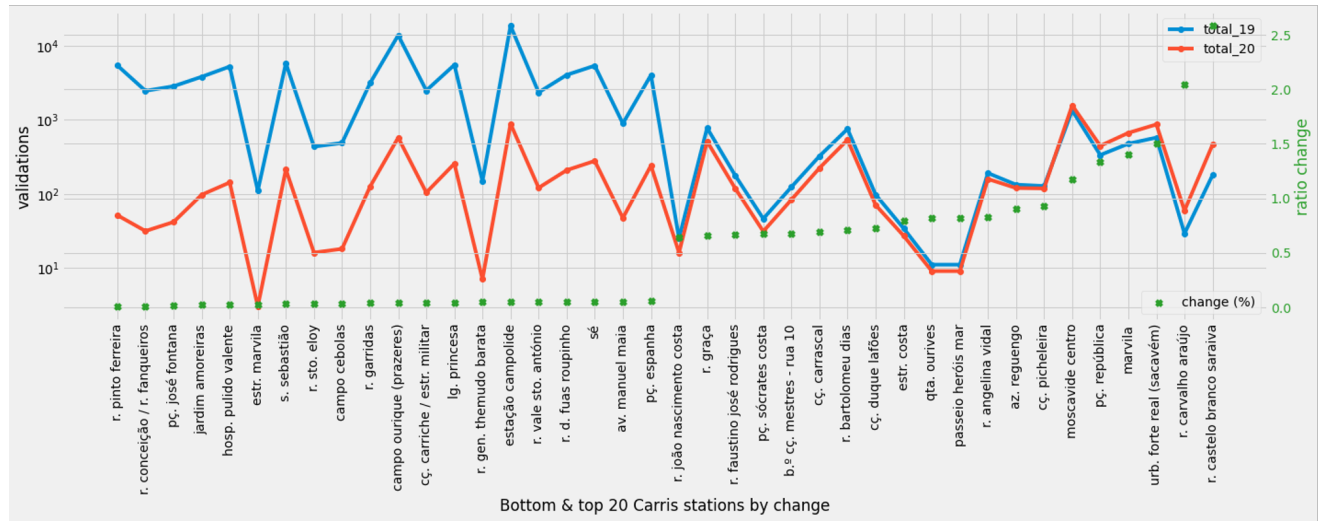

Figure 5. Demand for CARRIS stations in 2019 versus 2020 and ratio, top and bottom 20 stations.

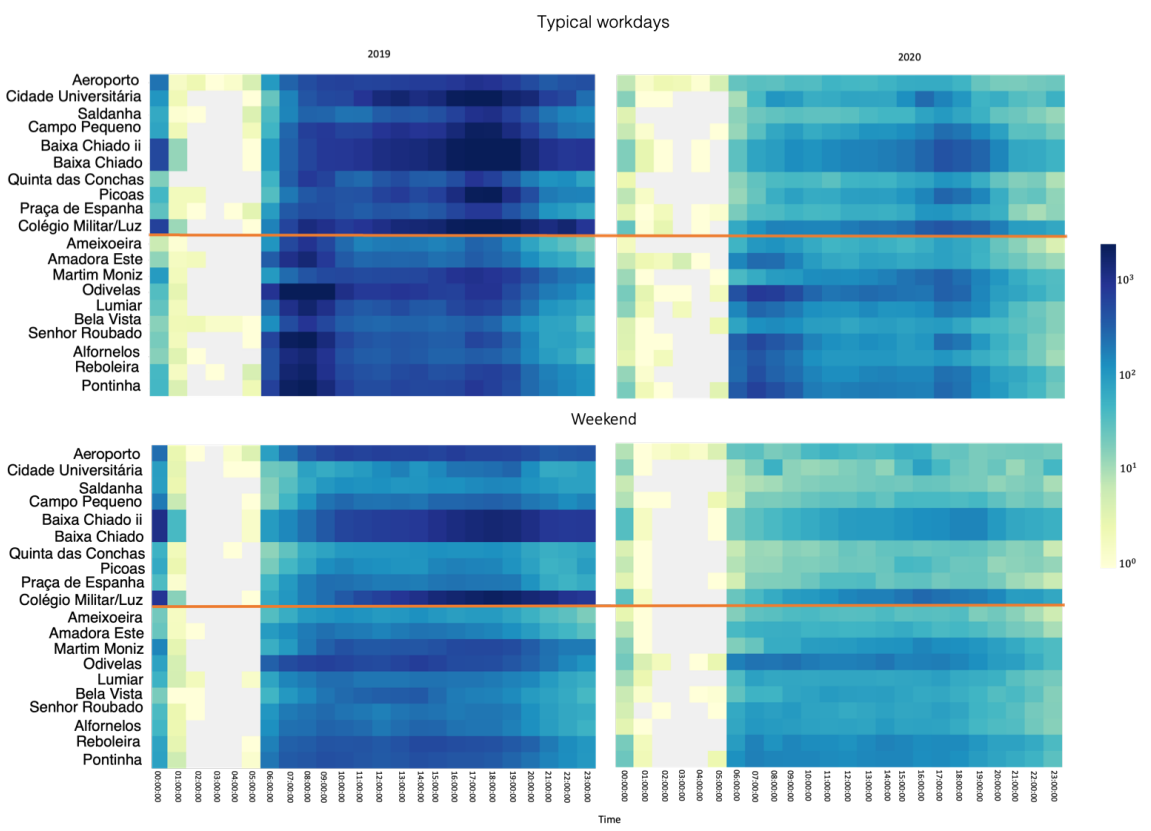

Figure 6. Demand for METRO stations during Tuesdays, Wednesdays and Thursdays (above) Weekend (bellow) by hour, top and bottom 10 stations by change from 2019 to 2020.

Considering the subway demand (Figure 6), we identify clear demand peaks from 7 a.m. to 10 a.m. and 4 p.m. to 8 p.m. corresponding to rush hours. Morning demand distribution approaches a normal hourly distribution, while the hourly demand distribution in the afternoon shows a skew towards earlier hours. Most of the stations on the top 10 (highest change in demand) are stations within the city center. The contraction in demand along these stations is possibly explained by a decrease in tourism activity and on-site commutes to business districts and schools. On the bottom of each heatmap we find the stations with least change-Ameixoeira, Amadora Este, Martim Moniz, Odivelas, Lumiar, Bela Vista, Senhor Roubado, Alfornelos, Reboleira and Pontinha - mainly residential areas. Along these stations, we observe an understandable symmetric pattern, a higher demand for entries during the morning period than the afternoon. 


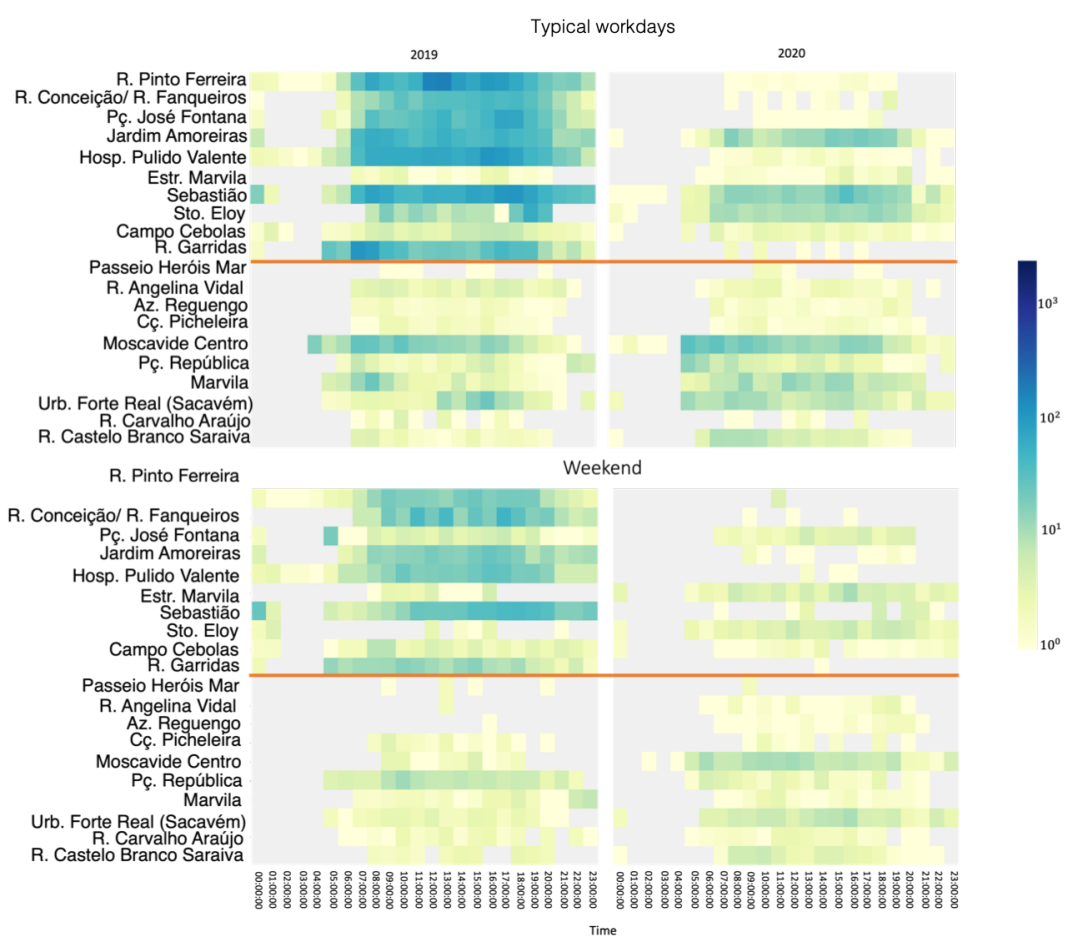

Figure 7. Demand for CARRIS stations during Tuesdays Wednesdays and Thursdays (above) Weekend (bellow) by hour, top and bottom 10 stations by change from 2019 to 2020.

The corresponding results for the bus-tram network are provided in Figure 7. There is a considerable difference in terms of average values through all the stops in the weekdays versus weekend. This can be noticed by looking at the scale of both heatmaps. The demand changes along the CARRIS stops follows a less delineate distribution, with no clear rush hours. We can observe an almost desertification effect on five of the top ten stops with most demand change. Different factors may cause this decrease, ranging from remote working and scholar programs to less-trivial factors, including decreased hospital visits and patient's mode preferences (Hosp. Pulido Valente stop serves a nearby hospital), and nonessential commerce (R. Conceição/R. Fanqueiros stop is known to serve a commercial zone in downtown Lisbon). Considering the stations with less diminishing effect, we see Moscavide Centro stop that showed an actual increase (compensating the significant decrease on the Moscavide subway station on the other side of the street). We can also observe delineate increases in demand over the early morning and afternoon peak hours, reinforcing the idea that the users using similar stops are usual daily commuters going and coming from the workplace. Other distinct features of each stop, including the properties of the associated routes and the surrounding area, may also play a pivotal role.

The proposed statistical and pattern-centric exploration of the changing mobility dynamics can be restricted to important city routes to assess vulnerabilities along the major city arteries. Figures 8 and 9 provide a a zoom over Figure 2, capturing the demand changes along the routes serving major city centres: Baixa (downtown), Marquês do Pombal, Saldanha, Sete Rios, Campo Grande, Oriente and Benfica. 


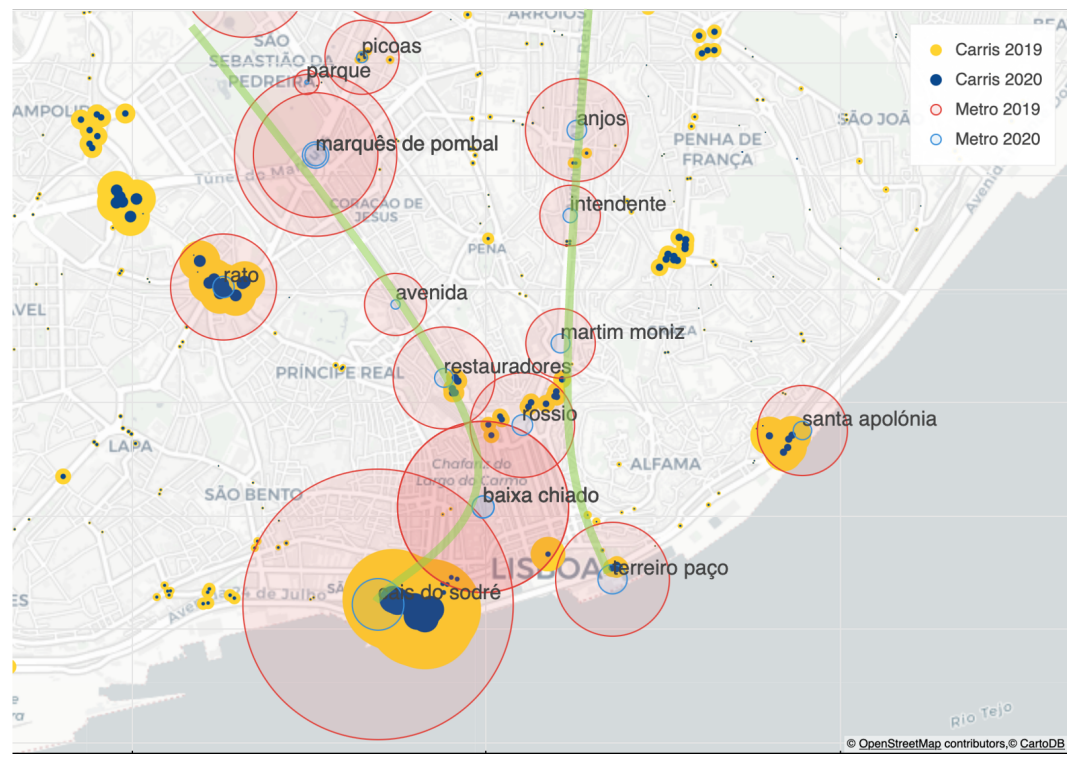

Figure 8. Station demand variation for 2019 vs. 2020 along Marques de Pombal to Baixa route.

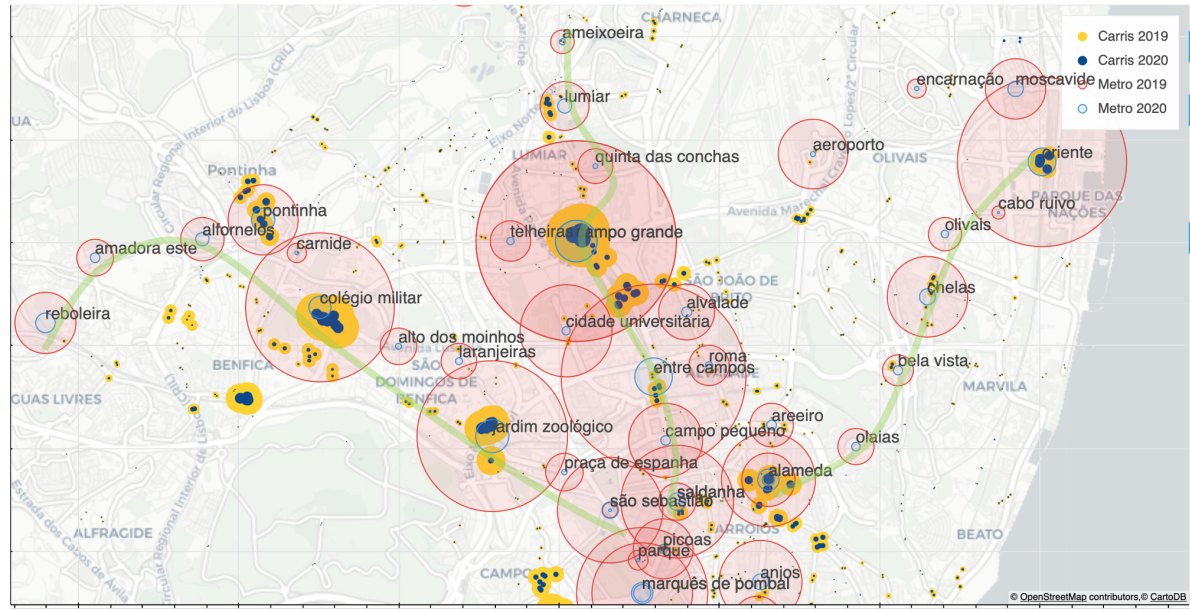

Figure 9. Station demand variation for 2019 vs. 2020 along three major routes from Marques de Pombal to Campo Grande, Benfica and Oriente routes.

\subsection{Passenger Demand Changes Using Actionable Pattern Discovery}

This section discusses the application of the proposed discriminative pattern discovery methodology (Section 4.3) to comprehensively find disruptions over order-preserving demand associations across geographies. As introduced, this approach allows us to trace statistically significant changes on the urban mobility dynamics throughout the different modes of transportation between two periods. Over 100 patterns satisfying the inputted discriminative power threshold were found with a median of four stations per pattern. Figures 10 and 11 illustrate some of the found biclusters, representative of the kinds of the discriminative patterns that were found using the order-preserving coherence assumption. Henriques et al. [47] statistical tests were applied to assess the significance of the found patterns. Each opaque line from a pattern, $\pi \Rightarrow t$, corresponds to a day supporting the discriminated period $t$, either yielding a blue or red shade depending on whether the pattern is discriminative of the 2019 or 2020 period, respectively. Pale lines correspond to the days of the counter period. Note that some of the plotted opaque lines may be hidden due to overlapping demands, a common occurrence for patterns with delineate statistical significance (low $p$-values). Demand is normalized to facilitate the analysis of preserved/disrupted orderings. 

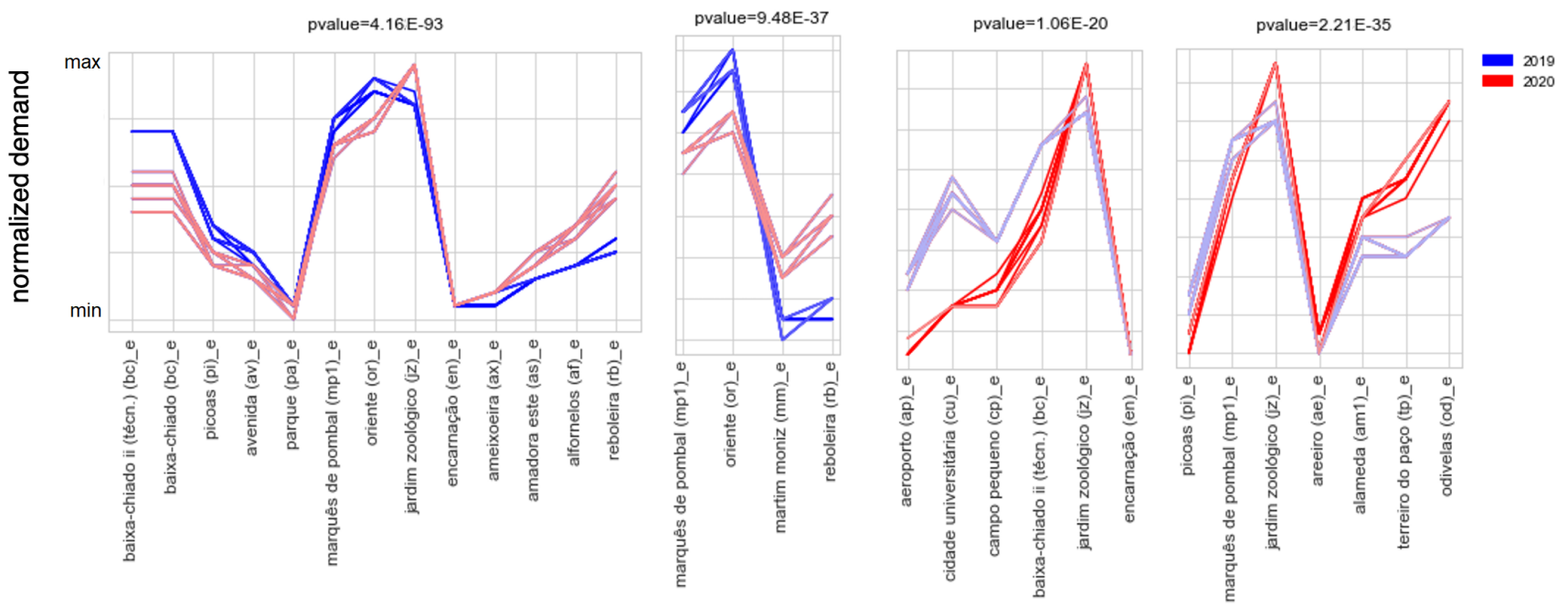

Figure 10. Illustrative order-preserving demand patterns found along the subway network.
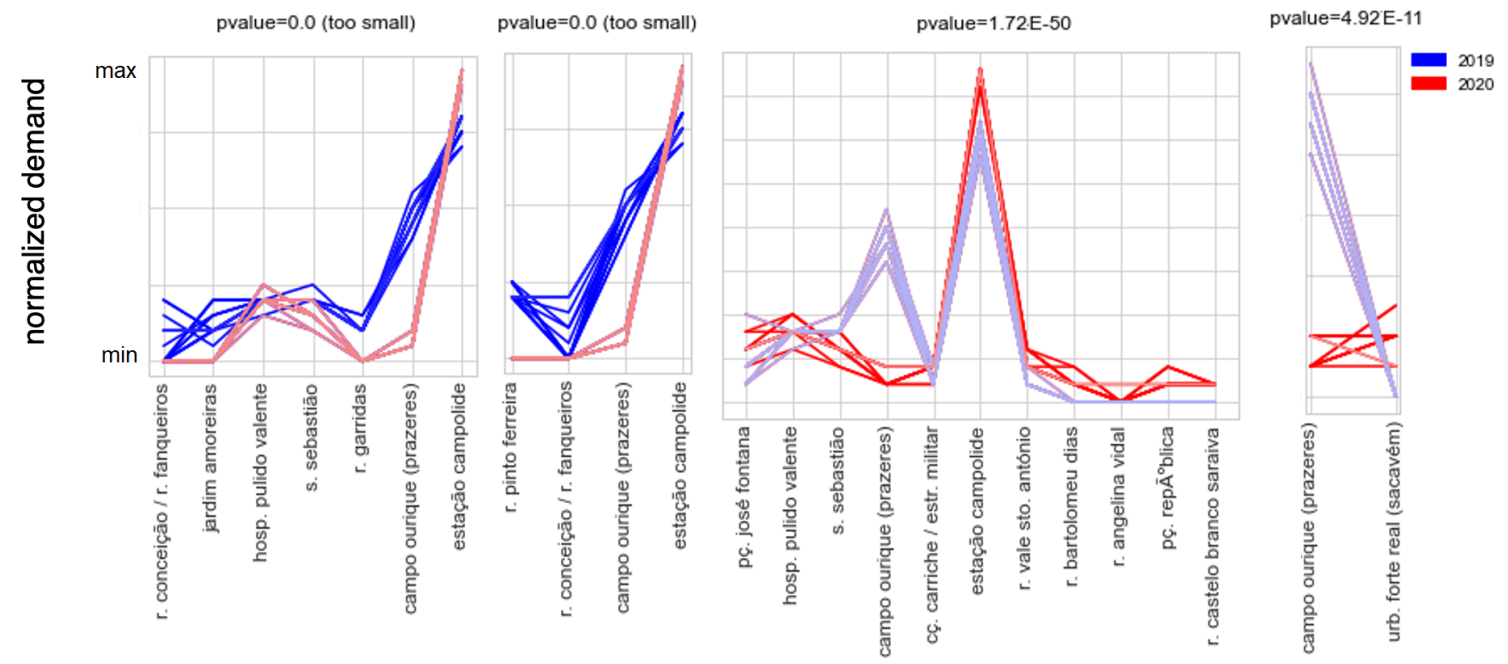

Figure 11. Illustrative order-preserving demand patterns found along the bus-tram network.

Considering the illustrative patterns found from the METRO network (Figure 10), we can observe that frequent orders of demand across stations for a given period can be disrupted on the counter period. Considering the first pattern, stations such as Jardim Zoológico corrupt the observed order-preserving demand. The increased normalized demand along Amadora Este, Alfornelos and Reboleira and the decrease along Baixa Chiado, Picoas and Avenida is also notorious. This pattern confirms our previous hypotheses that the subway routes serving peripheral and more residential areas are less affected by the pandemic, in contrast with central, business and commercial districts. Such pattern is also actionable, as the quantified disruptions to the expected demand across geographies can aid the planning of routes and vehicle frequency. The second bicluster shows a pattern, unlike the previous pattern, shows coherent orderings (low discriminative pattern) yet the observed demand differs in magnitude between 2019 and 2020 due to higher demand contractions observed in Marques de Pombal and Oriente against Maritm Moniz and Reboleira. The third and fourth pattern changes are descriminative for 2020. Aeurporto, Cidade Universitária, Campo Pequeno and Baixa Chiado have higher demand contraction while the residential Odivelas district is less impacted. These discriminative patterns of 2020 mobility dynamics are coherent with the insights gathered from the previous ones, offering further validation ground.

The analysis of the discriminative patterns from the passenger trip data at CARRIS exhibits statistically significant associations as well (see Figure 11). The first two illustrative 
patterns are discriminative of the pre-pandemic period (2019), with Jardim Amoreiras, R. Garridas, Campo de Ourique and R. Pinto Ferreira showing greater contraction in the normalized demand, and Estação Campolide the opposite trend. Considering the third and fourth patterns, we observe delineate disruptions to the expected orders caused by heightened changes in demand observed in Campo de Ourique and S. Sebastião stops, showing pandemic-specific divergences that should be carefully accounted at actical planning level.

\section{Discussion}

The reported research applied an enhanced methodology for discovering changing traffic patterns in public transportation systems using the city of Lisbon as a case study. More specifically, it bridged principles from urban computing and artificial intelligence algorithms to detect order-preserving patterns of demand among stations, along with their discriminative assessment to place a focus on actionable mobility changes. Principles from incremental data mining and online learning can be further placed to guarantee the ability to learn from continuously arriving trip record data. These principles guarantee the updatability of the computational models in the presence of more recent data, offering the possibility to dynamically reflect ongoing mobility changes throughout the current COVID-19 and future pandemics.

To the best of our knowledge, this is the first study that applied advanced pattern mining principles based on order-preserving biclustering algorithms within the urban mobility field. This feature enables transportation planners to analyse critical nodes using and holistic perspective along corridors or specific zones and trace changes occurring in different modes covering multiple geographies at a time. This approach aims to support the city and its public transportation operators to optimize supply, explore synergies and redesign dynamically their services and routes during disruptive events to better meet passenger needs.

Among the multiplicity of findings, the research revealed that the impact of COVID-19 on public transportation demand were considerably lower in those stations located in areas outside of Lisbon municipality and in zones with lower incomes. A possible interpretation is the need experienced by some daily commuters to continue relying on these transport modes to travel to work. The observed relationship between the demand impact and average income is cohesive with the arguments for inequality reported by Sharifi and Khavarian-Garmsird [50]. In this context, public transportation reveals to be key to address mobility needs during disruption and, hence, provision of safe services is a means to contribute to social equity goals. On the other hand, patterns and stations registering the highest decrease in demand are mostly located in specific areas in the city of Lisbon (higher price of housing per square meter) which seem to indicate that residents could adapt their working status and change it to digital forms. Overall, impacts of COVID-19 on public transportation highlighted the socioeconomic disparities of users across different areas and corridors.

The analysis of the changing mobility dynamics along the major city arteries (Figures 8 and 9) show that stations serving the downtown district (Cais do Sodré, Rossio and Terreiro do Paço) and the central business district (Saldanha and Marques de Pombal) suffered a heightened demand contraction after the pandemic, altering the pre-pandemic established demand effects of those classic traffic attracting poles. Upper regions of the Avenida Almirante Reis also entailed higher changes when compared with stations lower in the same avenue, for both modes of transportation. On the Marques de Pombal to Campo Grande route, we observe a greater impact on the demand for METRO stations over bus stops, particular those stations serving large commercial and sports poles, such as Benfica and Campo Grande. The observed degree of pandemic-triggered changes to urban mobility are consistent with other works $[27,30,51,52]$.

The gathered discriminative patterns (Figures 10 and 11) augment these views, supporting the comparison of the relative geographical variations on the traffic demand 
with the aim of unveiling actionable bottlenecks. As the rates of change in demand are not uniform throughout the city, the discovery of patterns with such actionable characteristics are essential to produce focal information for promoting more resilient public transportation system.

Further policy and planning measures are expected to envisage a roadmap or pathway for a sustainable and multimodal mobility within Lisbon metropolitan area. In future work, it would be also important to understand if these patterns found have changed after the second and third waves of the COVID-19 pandemic in Lisbon. The computational approach can be directly applied to periods other than those considered, including different moments within the pandemic. Following the methodology introduced, the user only has to specify the periods of interest, with the patterns and views being dynamically updated.

Author Contributions: All authors contributed to the design, writing and agreed to the published version of the manuscript. All authors have read and agreed to the published version of the manuscript.

Funding: The authors thank CARRIS, METRO and Câmara Municipal de Lisboa (Gabinete de Mobilidade and Centro de Operações Integrado) for the provision of data, support and valuable feedback. This work was further supported by national funds through Fundação para a Ciência e Tecnologia (FCT) under project ILU (DSAIPA/DS/0111/2018) and INESC-ID pluriannual (UIDB/50021/2020).

Institutional Review Board Statement: Not applicable.

Informed Consent Statement: Not applicable.

Data Availability Statement: Passenger trip record data are not publicly available due to privacy concerns. Urban planning and GTFS data available at Lisboa Aberta http:/ / dados.cm-lisboa.pt/.

Conflicts of Interest: The authors declare no conflict of interest.

\begin{tabular}{|c|c|}
\hline \multicolumn{2}{|c|}{ Abbreviations } \\
\hline BicPAMS & Biclustering Pattern Mining-based Software \\
\hline CARRIS & Companhia Carris de Ferro de Lisboa \\
\hline GTFS & General Transit Feed Specification \\
\hline METRO & Metropolitano de Lisboa \\
\hline
\end{tabular}

\section{References}

1. De Vos, J. The effect of COVID-19 and subsequent social distancing on travel behavior. Transp. Res. Interdiscip. Perspect. 2020, $5,100121$.

2. Zheng, Y.; Capra, L.; Wolfson, O.; Yang, H. Urban computing: Concepts, methodologies, and applications. ACM Trans. Intell. Syst. Technol. (TIST) 2014, 5, 1-55. [CrossRef]

3. Tuchen, S. Multimodal Transportation Operational Scenario And Conceptual Data Model For Integration With Uam. In Proceedings of the 2020 Integrated Communications Navigation and Surveillance Conference (ICNS), Herndon, VA, USA, 8-10 September 2020. [CrossRef]

4. Jenelius, E.; Cebecauer, M. Impacts of COVID-19 on public transport ridership in Sweden: Analysis of ticket validations, sales and passenger counts. Transp. Res. Interdiscip. Perspect. 2020, 8, 100242.

5. Neves, F.; Finamore, A.; Henriques, R. Efficient discovery of emerging patterns in heterogeneous spatiotemporal data from mobile sensors. In Proceedings of the EAI International Conference on Mobile and Ubiquitous Systems: Computing, Networking and Services, Beppu, Japan, 7-9 December 2020; pp. 1-10. [CrossRef]

6. Lemonde, C.; Arsénio, E.; Henriques, R. Exploring Multimodal Mobility Patterns With Big Data in the City of Lisbon. In Proceedings of the European Transport Conference 2020, Milan, Italy, 9-11 September 2020. [CrossRef]

7. Cai, P.; Wang, Y.; Lu, G.; Chen, P.; Ding, C.; Sun, J. A spatiotemporal correlative k-nearest neighbor model for short-term traffic multistep forecasting. Transp. Res. Part C Emerg. Technol. 2016, 62, 21-34. [CrossRef] [PubMed]

8. Henriques, R.; Antunes, C.; Madeira, S.C. Generative modeling of repositories of health records for predictive tasks. Data Min. Knowl. Discov. 2015, 29, 999-1032. [CrossRef]

9. Wu, C.W.; Lin, Y.F.; Yu, P.S.; Tseng, V.S. Mining high utility episodes in complex event sequences. In Proceedings of the 19th ACM SIGKDD International Conference on Knowledge Discovery and Data Mining, Chicago, IL, USA, 11-14 August 2013; pp. 536-544. [CrossRef]

10. Dabiri, S.; Heaslip, K. Developing a Twitter-based traffic event detection model using deep learning architectures. Expert Syst. Appl. 2019, 118, 425-439. [CrossRef] [PubMed] 
11. Neves, F.; Finamore, A.C.; Madeira, S.C.; Henriques, R. Mining Actionable Patterns of Road Mobility From Heterogeneous Traffic Data Using Biclustering. IEEE Trans. Intell. Transp. Syst. 2021. [CrossRef]

12. Dong, G.; Li, J. Efficient mining of emerging patterns: Discovering trends and differences. In Proceedings of the Fifth ACM SIGKDD International Conference on Knowledge Discovery and Data Mining, San Diego, CA, USA, 15-18 August 1999; pp. 43-52. [CrossRef]

13. Salamanis, A.; Margaritis, G.; Kehagias, D.D.; Matzoulas, G.; Tzovaras, D. Identifying patterns under both normal and abnormal traffic conditions for short-term traffic prediction. Transp. Res. Procedia 2017, 22, 665-674. [CrossRef]

14. Rodrigues, F.; Borysov, S.S.; Ribeiro, B.; Pereira, F.C. A Bayesian Additive Model for Understanding Public Transport Usage in Special Events. IEEE Trans. Pattern Anal. Mach. Intell. 2017, 39, 2113-2126. [CrossRef]

15. Liao, Y.; Gil, J.; Pereira, R.H.; Yeh, S.; Verendel, V. Disparities in travel times between car and transit: Spatiotemporal patterns in cities. Sci. Rep. 2020, 10, 4056. [CrossRef]

16. Guo, D.; Zhu, X.; Jin, H.; Gao, P.; Andris, C. Discovering Spatial Patterns in Origin-Destination Mobility Data. Trans. GIS 2012, 16, 411-429. [CrossRef]

17. Li, L.; Jiang, R.; He, Z.; Chen, X.M.; Zhou, X. Trajectory data-based traffic flow studies: A revisit. Transp. Res. Part C Emerg. Technol. 2020, 114, 225-240. [CrossRef]

18. Gonzalez, M.C.; Hidalgo, C.A.; Barabasi, A.L. Understanding individual human mobility patterns. Nature 2008, 453, 779-782.

19. Hasan, S.; Schneider, C.; Ukkusuri, S.; Gonzalez, M.C. Spatiotemporal Patterns of Urban Human Mobility. J. Stat. Phys. 2012, 151, 1-15. [CrossRef]

20. Necula, E. Analyzing traffic patterns on street segments based on GPS data using R. Transp. Res. Procedia 2015, 10, 276-285. [CrossRef]

21. Rempe, F.; Huber, G.; Bogenberger, K. Spatio-Temporal Congestion Patterns in Urban Traffic Networks. Transp. Res. Procedia 2016, 15, 513-524. [CrossRef]

22. Song, J.; Zhao, C.; Zhong, S.; Nielsen, T.A.S.; Prishchepov, A.V. Mapping spatio-temporal patterns and detecting the factors of traffic congestion with multi-source data fusion and mining techniques. Comput. Environ. Urban Syst. 2019, 77, 101364. [CrossRef]

23. Giannotti, F.; Nanni, M.; Pinelli, F.; Pedreschi, D. Trajectory pattern mining. In Proceedings of the 13th ACM SIGKDD International Conference on Knowledge Discovery and Data Mining, San Jose, CA, USA, 12-15 August 2007; pp. 330-339. [CrossRef]

24. Inoue, R.; Miyashita, A.; Sugita, M. Mining spatio-temporal patterns of congested traffic in urban areas from traffic sensor data. In Proceedings of the 2016 IEEE 19th International Conference on Intelligent Transportation Systems (ITSC), Rio de Janeiro, Brazil, 1-4 November 2016; pp. 731-736. [CrossRef]

25. Chen, Z.; Yang, Y.; Huang, L.; Wang, E.; Li, D. Discovering Urban Traffic Congestion Propagation Patterns With Taxi Trajectory Data. IEEE Access 2018, 6, 69481-69491. [CrossRef]

26. Yang, J.; Zhang, X.; Qiao, Y.; Fadlullah, Z.; Kato, N. Global and individual mobility pattern discovery based on hotspots. In Proceedings of the 2015 IEEE International Conference on Communications (ICC), London, UK, 8-12 June 2015; pp. 5577-5582.

27. Aloi, A.; Alonso, B.; Benavente, J.; Cordera, R.; Echániz, E.; González, F.; Ladisa, C.; Lezama-Romanelli, R.; López-Parra, Á.; Mazzei, V.; et al. Effects of the COVID-19 lockdown on urban mobility: Empirical evidence from the city of Santander (Spain). Sustainability 2020, 12, 3870. [CrossRef] [PubMed]

28. Aletta, F.; Brinchi, S.; Carrese, S.; Gemma, A.; Guattari, C.; Mannini, L.; Patella, S.M. Analysing urban traffic volumes and mapping noise emissions in Rome (Italy) in the context of containment measures for the COVID-19 disease. Noise Mapp. 2020, 7, 114-122. [PubMed]

29. Tian, X.; An, C.; Chen, Z.; Tian, Z. Assessing the impact of COVID-19 pandemic on urban transportation and air quality in Canada. Sci. Total Environ. 2021, 765, 144270.

30. Campisi, T.; Basbas, S.; Skoufas, A.; Akgün, N.; Ticali, D.; Tesoriere, G. The Impact of COVID-19 Pandemic on the Resilience of Sustainable Mobility in Sicily. Sustainability 2020, 12, 8829.

31. Przybylowski, A.; Stelmak, S.; Suchanek, M. Mobility Behaviour in View of the Impact of the COVID-19 Pandemic-Public Transport Users in Gdansk Case Study. Sustainability 2021, 13, 364. [CrossRef]

32. Tamagusko, T.; Ferreira, A. Data-Driven Approach to Understand the Mobility Patterns of the Portuguese Population during the COVID-19 Pandemic. Sustainability 2020, 12, 9775. [CrossRef]

33. Gokcan, S. Forecasting volatility of emerging stock markets: Linear versus non-linear GARCH models. J. Forecast. 2000, 19, 499-504.

34. Franses, P.H.; Van Dijk, D. Non-Linear Time Series Models in Empirical Finance; Cambridge University Press: Cambridge, UK, 2000. [CrossRef]

35. Li, J.; Wong, L. Identifying good diagnostic gene groups from gene expression profiles using the concept of emerging patterns. Bioinformatics 2002, 18, 725-734. [CrossRef]

36. Liu, H.; Li, J.; Wong, L. A comparative study on feature selection and classification methods using gene expression profiles and proteomic patterns. Genome Inform. 2002, 13, 51-60. [CrossRef]

37. Novak, P.K.; Lavrač, N.; Webb, G.I. Supervised descriptive rule discovery: A unifying survey of contrast set, emerging pattern and subgroup mining. J. Mach. Learn. Res. 2009, 10, 377-403. 
38. Wu, R.C.; Chen, R.S.; Chen, C.C. Data mining application in customer relationship management of credit card business. In Proceedings of the 29th Annual International Computer Software and Applications Conference (COMPSAC'05), Edinburgh, UK, 26-28 July 2005; Volume 2, pp. 39-40. [CrossRef]

39. Song, H.S.; kyeong Kim, J.; Kim, S.H. Mining the change of customer behavior in an internet shopping mall. Expert Syst. Appl. 2001, 21, 157-168. [CrossRef]

40. Henriques, R.; Madeira, S.C. Triclustering algorithms for three-dimensional data analysis: A comprehensive survey. ACM Comput. Surv. (CSUR) 2018, 51, 1-43. [CrossRef] [PubMed]

41. Henriques, R.; Antunes, C. Learning predictive models from integrated healthcare data: Extending pattern-based and generative models to capture temporal and cross-attribute dependencies. In Proceedings of the 2014 47th Hawaii International Conference on System Sciences, Waikoloa, HI, USA, 6-9 January 2014; pp. 2562-2569.

42. Vico, A.M.G.; Carmona, C.; Gonzalez, P.; Seker, H.; Del Jesus, M.J. FEPDS: A Proposal for the Extraction of Fuzzy Emerging Patterns in Data Streams. IEEE Trans. Fuzzy Syst. 2020. [CrossRef] [PubMed]

43. Henriques, R.; Antunes, C.; Madeira, S.C. A Structured View on Pattern Mining-based Biclustering. Pattern Recognit. 2015, 4, 3941-3958. [CrossRef]

44. Henriques, R.; Madeira, S. BicPAM: Pattern-based biclustering for biomedical data analysis. Alg. Mol. Biol. 2014, 9, 27. [CrossRef]

45. Henriques, R.; Ferreira, F.L.; Madeira, S.C. BicPAMS: Software for biological data analysis with pattern-based biclustering. BMC Bioinform. 2017, 18, 82.

46. Henriques, R.; Madeira, S.C. BicSPAM: Flexible biclustering using sequential patterns. BMC Bioinform. 2014, 15, 130.

47. Henriques, R.; Madeira, S.C. BSig: Evaluating the statistical significance of biclustering solutions. Data Min. Knowl. Discov. 2018, 32, 124-161.

48. Madeira, S.C.; Oliveira, A.L. Biclustering Algorithms for Biological Data Analysis: A Survey. IEEE/ACM Trans. Comput. Biol. Bioinform. 2004, 1, 24-45.

49. Statistics Portugal-Web Portal. Gross Reported Income Per Tax Household (€) by Geographic Localization. Available online: https: / / www.ine.pt/xportal/xmain?xpid=INE\&xpgid=ine_indicadores\&indOcorrCod=0009762\&contexto=bd\&selTab= tab2. (accessed on 24 June 2012).

50. Sharifi, A.; Khavarian-Garmsir, A.R. The COVID-19 pandemic: Impacts on cities and major lessons for urban planning, design, and management. Sci. Total Environ. 2020, 749, 142391.

51. Hadjidemetriou, G.M.; Sasidharan, M.; Kouyialis, G.; Parlikad, A.K. The impact of government measures and human mobility trend on COVID-19 related deaths in the UK. Transp. Res. Interdiscip. Perspect. 2020, 6, 100167.

52. Bucsky, P. Modal share changes due to COVID-19: The case of Budapest. Transp. Res. Interdiscip. Perspect. 2020, 8, 100141. 